\title{
Desarrollo de frondes circinados y valor nutricional de helechos alimenticios en el Santuario Nacional Ampay-Apurímac
}

\section{Development of circinated fronds and nutritional value of food ferns in the Ampay-Apurímac National Sanctuary}

\section{René Farfán Zegarra ${ }^{1,3}$, Carlos E. Coacalla Castillo ${ }^{2}$, Gladys M. Castro Peréz ${ }^{2}$, Maria E. Holgado Rojas ${ }^{1,3}$, Ruth Lazarte Lovaton ${ }^{2,3}$ \& Daniel Paucarmayta Holgado ${ }^{2}$}

\author{
${ }^{1}$ Universidad Nacional de San Antonio Abad del Cusco. Sociedad Botánica del Cusco. Av. de la ${ }^{2}$ Cultura 733 \\ Cusco,Perú.rfziva22@gmail.com \\ 2Universidad Nacional Micaela Bastidas de Apurímac, Av. Inca Garcilazo de laVega S/N,Abancay \\ ${ }^{3}$ Sociedad Botánica del Cusco. Av. de La Cultura 773, Cusco.
}

\section{Resumen}

El helecho Asplenium squamosum que crece en el Santuario Nacional del Ampay Abancay - Apurímac y el distrito de Lamay, Calca - Cusco, con importancia económica por ser considerados ornamentales, pero con potencial alimenticio y medicinal para los pobladores locales. Tiene como propósito determinar el crecimiento de frondes circinados, siendo un estudio transversal, de diseño correlacional y descriptivo. El crecimiento óptimo de las frondes se observó durante los meses enero y febrero con longitudes que alcanzaban de 18 a $27 \mathrm{~cm}$, ello por la influencia de la precipitación pluvial promedio anual $50.72 \mathrm{~mm}$; temperatura promedio anual $15.05^{\circ} \mathrm{C}$ y humedad relativa $73 \%$. La frecuencia de frondes circinados por cada mata varía de 1 a 7 , siendo el promedio de 4 unidades por cada planta de helecho. El Coeficiente de correlación exponencial de longitud y peso de las frondes fue $(\mathrm{r}=0.90)$. El análisis nutricional en cuanto a proteínas y fibras fueron mayores a $3 \%$, carbohidratos $4.36 \%$. Se halló un alto contenido de Potasio, Fósforo, Calcio y Magnesio.

Palabras clave. Asplenium squamosum, Frondes circinados, Helecho, Santuario Nacional de Ampay, Valor nutricional.

Abstract

The Asplenium squamosum fern that grows in the National Sanctuary of Ampay Abancay - Apurímac and the district of Lamay, Calca - Cusco, with economic importance for being considered ornamental, but with potential use as food and medicine for local people. Its purpose is to determine the growth of circinate fronds, being a cross-sectional study, with a correlational and descriptive design. The optimal growth of the fronds was observed during the months of January and February with lengths that reach 18 to $27 \mathrm{~cm}$, that is, due to the influence of the annual average rainfall of $50.72 \mathrm{~mm}$ and the average annual temperature of $15.05^{\circ} \mathrm{C}$ and $73 \%$ relative humidity. The frequency of circinate fronds for each bush varies from 1 to 7 , the average being 4 units per fern plant. The exponential correlation coefficient of length and weight of the fronds was $(r=0.90)$. The nutritional analysis in terms of proteins and fibers were greater than $3 \%$, carbohydrates 4.36\%, a high content of Potassium, Phosphorus, Calcium and Magnesium was found.

KeyWords. Aspenium squamosum, Fern, Fronds circinate, National Sanctuary of Ampay, Nutritional value

\section{Introducción}

Los helechos integran un grupo de plantas vasculares especialmente vulnerables a los- cambios climáticos, la deforestación, al avance de la frontera agropecuaria, a los incendios y a la extracción para su comercialización (Cabrera, 1976; Brown et al., 2006). 
Las informaciones acerca de la importancia económica de los helechos indican principalmente su empleo como plantas ornamentales y en menor medida, como alimenticias y medicinales. Varias especies de helechos han sido reportados como alimenticios tanto en las regiones de Apurímac y Cusco, destacando principalmente Asplenium (Apurímac) y Dennstaedtia (Cusco).

La lámina foliar madura de Asplenium s. L. es varias veces dividida y glabra, raramente pubescente y coriácea. La planta crece naturalmente en el interior del bosque húmedo del Santuario Nacional de Ampay. El brote de las frondes se inicia en el mes de noviembre de cada año y termina en marzo o abril, luego, las frondas se abren para convertirse en hojas maduras. La familia Aspleniaceae fue estudiada por varios autores, especialmente desde el punto de vista de su distribución mundial (Mickel\& Smith, 2004), (Tryon \& Tryon, 1982). La distribución en América del Sur y Central del Asplenium s. L. fueron investigados por (Tryon \& Stolze, 1993), (Akirov, 2009), (Candollea, 2007) y (Navarrete, 2006); según los autores la especie crece en Brasil, Bolivia, Colombia, Costa Rica, Ecuador, Haití, Venezuela, Argentina y Perú.

Especies de esta familia fueron registradas en Apurímac en la provincia de Abancay distrito de Tamburco, lugar Santuario Nacional de Ampay, que es una Unidad de Conservación creada el 23 de julio del 1987, con el propósito de proteger con carácter de intangible un rodal de bosque de Podocarpus glomeratus, "Intimpa" donde son utilizados como plantas alimenticias conocidos como "Ullpu" reportándose tres especímenes más: Asplenium fragans SW., Asplenium praemorsum SW y Asplenium sessilifolium Desv (Venero \&Tupayachi, 1989).
Las localidades de mayor producción del "ullpu" Asplenium squamosum Linnaeus, están al sur: Cusco en 16 sectores y Apurímac en 11 sectores, en los valores agregados se tiene en potajes con alto valor alimenticio del Dominio Andino del sur al norte del país (Pérez \& Arce, 2014). En el distrito de Lamay, departamento de Cusco, también se conoce como Ullpu a Dennstaedtia glauca (Cav.) C. Chr. ex Looser, que es utilizado en la alimentación en el distrito de Lamay en los meses de julio y agosto, donde los prófilos se encuentran en plena formación siendo estas la parte comestible y con el cual se prepara el "Ullpu Haucha” plato típico y central de la festividad de Santa Rosa patrona del distrito. También se usan los nombres ullpu y raki-raki para helechos de los géneros Asplenium s. L, Pteridium aquilinum y Dryopteris glandulosolanosa, cuyas partes comestibles siempre son los brotes tiernos antes de la apertura del botón foliar (prófilos). Al respecto es importante señalar que el ullpu es un alimento silvestre tradicional en el Valle Sagrado de los Incas, por su valor nutricional, además de ser una buena fuente de fibra y ser bajo en sodio, contienen vitaminas A y C, potasio, fósforo y hierro, también son una fuente de ácidos grasos y omega 3. Su valor antioxidante es alto (Agroeco, 2013). Los brotes de helechos se pueden comer blanqueados o hervidos, para servirlos en ensaladas o salteados con mantequilla. También se preparan en guisos, sopas, frituras, así como en distintas preparaciones chinas y japonesas (Alva, 2013), por lo que en la presente investigación se estudia el crecimiento de las frondes circinados en condiciones del bosque de Ampay, cuantificándolos por cada mata, así como el análisis de valores nutricionales de la especie. 


\section{Material y Métodos Área de estudio}

El área de estudio fue el bosque húmedo y nublado del Santuario Nacional del Ampay, distrito de Tamburco, provincia de Abancay Apurímac. La investigación se realizó en el mes de noviembre del 2013 a octubre del año del 2014, el ámbito de estudio comprende las altitudes de 2,750 a 3,500 msnm, cuyas coordenadas UTM corresponde a Latitud sur: $13^{\circ} 33^{\prime} 23^{\prime \prime}-13^{\circ} 26^{\prime} 46^{\prime \prime}$ y Longitud oeste: $72^{\circ} 55^{\prime} 52^{\prime \prime}-72^{\circ} 51^{\prime} 06^{\prime \prime}$. El S. H. de Ampay se encuentra en la zona de vida de bosque húmedo Montado SubTropical (bh-MST).

El Diseño consiste en elaborar el diagrama de esparcimiento y curva exponencial de la relación Longitud $(\mathrm{X})$ y peso $(\mathrm{Y})$ de frondes circinados de Asplenium s. $L$. Se ha aplicado el diseño correlacional. Se seleccionaron aleatoriamente 30 plantas de helecho que crecen naturalmente en los márgenes del riachuelo que alimenta a la laguna pequeña del Ampay "Anqasccocha”. Las observaciones y mediciones de crecimiento de frondes circinados se realizaron durante un año. Los datos de crecimiento fueron obtenidos mediante mediciones de longitud en la misma planta utilizando una wincha metálica. Los parámetros ambientales como la precipitación y temperatura fueron tomados de los instrumentos instalados en la Estación Meteorológica más cercana, ubicada en la Granja de San Antonio del Ministerio de Agricultura. Para obtener los datos de frecuencia de frondes circinados por cada mata o planta, se aplicó el conteo directo en 30 matas seleccionadas anteriormente.

La obtención de datos para el diagrama de esparcimiento y curva exponencial de la relación Longitud (X) y peso (Y) se realizó- -en el mes de febrero del año 2014, para lo que se recolectaron al azar, 30 nuevas muestras de frondes circinados, en las cercanías de los anteriores; las magnitudes longitud y peso fueron medidos con una regla metálica y una balanza electrónica digital en el laboratorio de Biología, por último, estas muestra fueron llevados al laboratorio de Química para la determinación de los macronutrientes y micronutrientes.

\section{Resultados}

En la figura 1 se reporta el desarrollo de frondes circinados de Asplenium s. L., iniciándose el crecimiento generalmente con las precipitaciones pluviales del mes de noviembre y diciembre, observándose el tamaño de los brotes en unos $04 \mathrm{~cm}$ de longitud. En los meses de enero y febrero se da un óptimo crecimiento de las frondes de 18 a $27 \mathrm{~cm}$ de longitud bajo la influencia de la precipitación pluvial promedio de $111.5 \mathrm{~mm}$ y temperatura promedio de unos $15.05^{\circ} \mathrm{C}$ y 73 $\%$ de humedad relativa. Entre marzo, abril y mayo en adelante, el crecimiento se hace mínimo en la etapa de maduración y envejecimiento de la lámina foliar, que puede alcanzar hasta más de $50 \mathrm{~cm}$ de longitud dependiendo de los factores ambientales.

En la figura 2 se observa las barras de la frecuencia de frondes circinados por cada planta o mata de helecho, que varía de 1 a 7 , siendo en promedio 4 unidades de frondas por cada planta de Asplenium s. L.

En la figura 3 el diagrama de esparcimiento y curva de regresión exponencial de la relación de longitud y peso de frondes circinados, calculado a partir de la función exponencial de la forma $\mathrm{Y}^{*}=\mathrm{ab}^{\mathrm{x}}$, de valores $\mathrm{a}=0.56 \mathrm{yb}=$ $(0.11)^{x}$. 


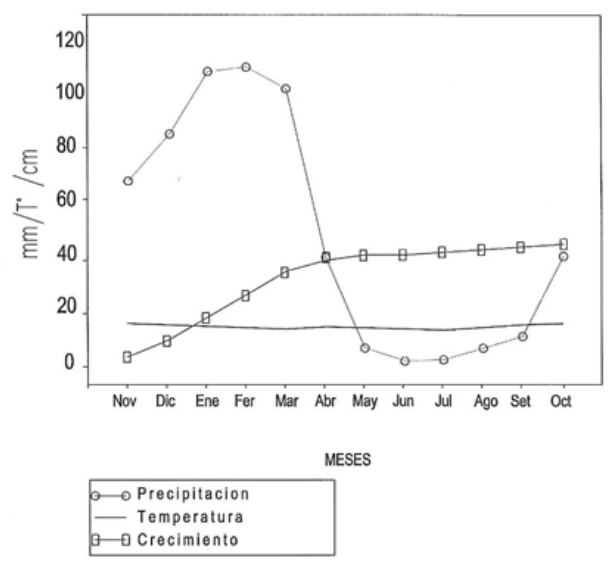

Figura 1. Crecimiento de frondes circinados de Asplenium squamosum $L$. en el curso del año y su relación con la precipitación pluvial y temperatura ambiental.

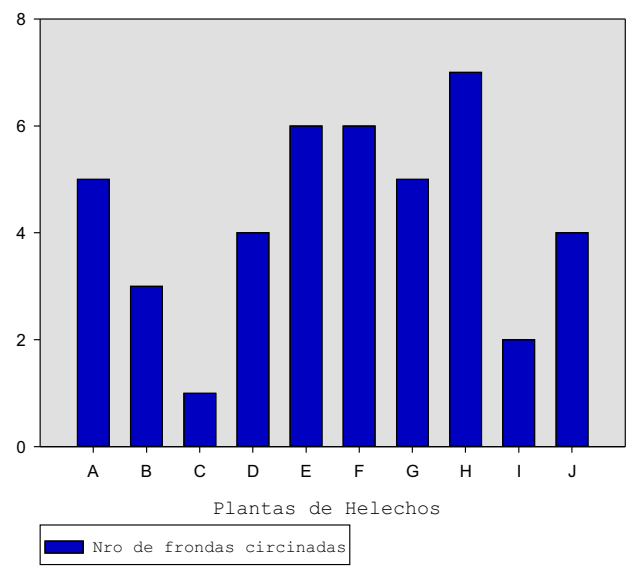

Figura 2. Frecuencia de frondes circinados por mata o planta.

El coeficiente de correlación exponencial $r$ $=0.90$, es la tasa de crecimiento de las frondes en un período corto de cuatro primeros meses (noviembre, diciembre, enero y febrero) donde se observó el aumento proporcional entre los variables longitud y peso. Coeficiente de determinación: $0.90 * 100=90.3 \%$, indica que existe una fuerte influencia de la variable longitud sobre el peso.
Relacion Longitud/peso de Asplenium squamosum

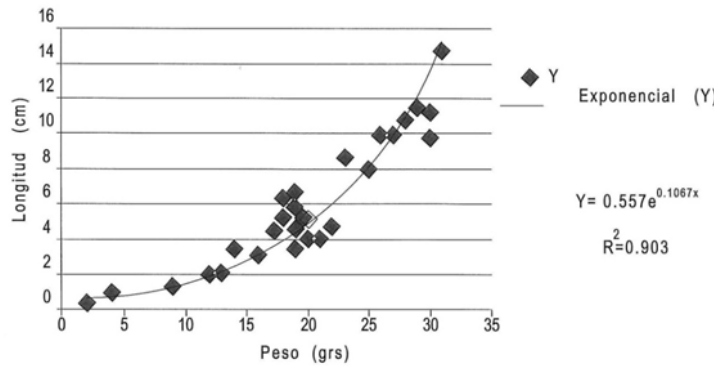

Figura 3. Diagrama de esparcimiento y curva exponencial de la relación Longitud $(\mathrm{X})$ y peso (Y) de frondes circinados de Asplenium squamosum $L$. extraído del bosque de Ampay.

Tabla 1. Análisis químicos de nutrientes de frondes circinados de Asplenium squamosum $L$. "Ullpu" deAmpay.

\begin{tabular}{lr}
\hline Analisis proximal & Valores \\
\hline & \\
Humedad \% & 90.80 \\
Proteinas \% & 3.25 \\
Grasa \% & 0.78 \\
Ceniza & 0.81 \\
Fibra \% & 3.96 \\
Carbohidratos \% & 4.36 \\
Calcio (mg/100) & 32.00 \\
Fosforo (mg/100) & 46.00 \\
Hierro (mg/100)0 & .94 \\
Magnesio (mg/100 & 21.00 \\
Potasio (mg/100) & 350.00 \\
Zinc (mg/100) & 0.12 \\
Cromo (mg/100) & 0.004 \\
Cobre (mg/100) & 0.007 \\
\hline
\end{tabular}

\section{Discusión}

El crecimiento es producto de la división celular y la expansión de las células que son las dos formas en que crecen los helechos.

La división celular crea células adicionales, mientras que la expansión de células crea un- 


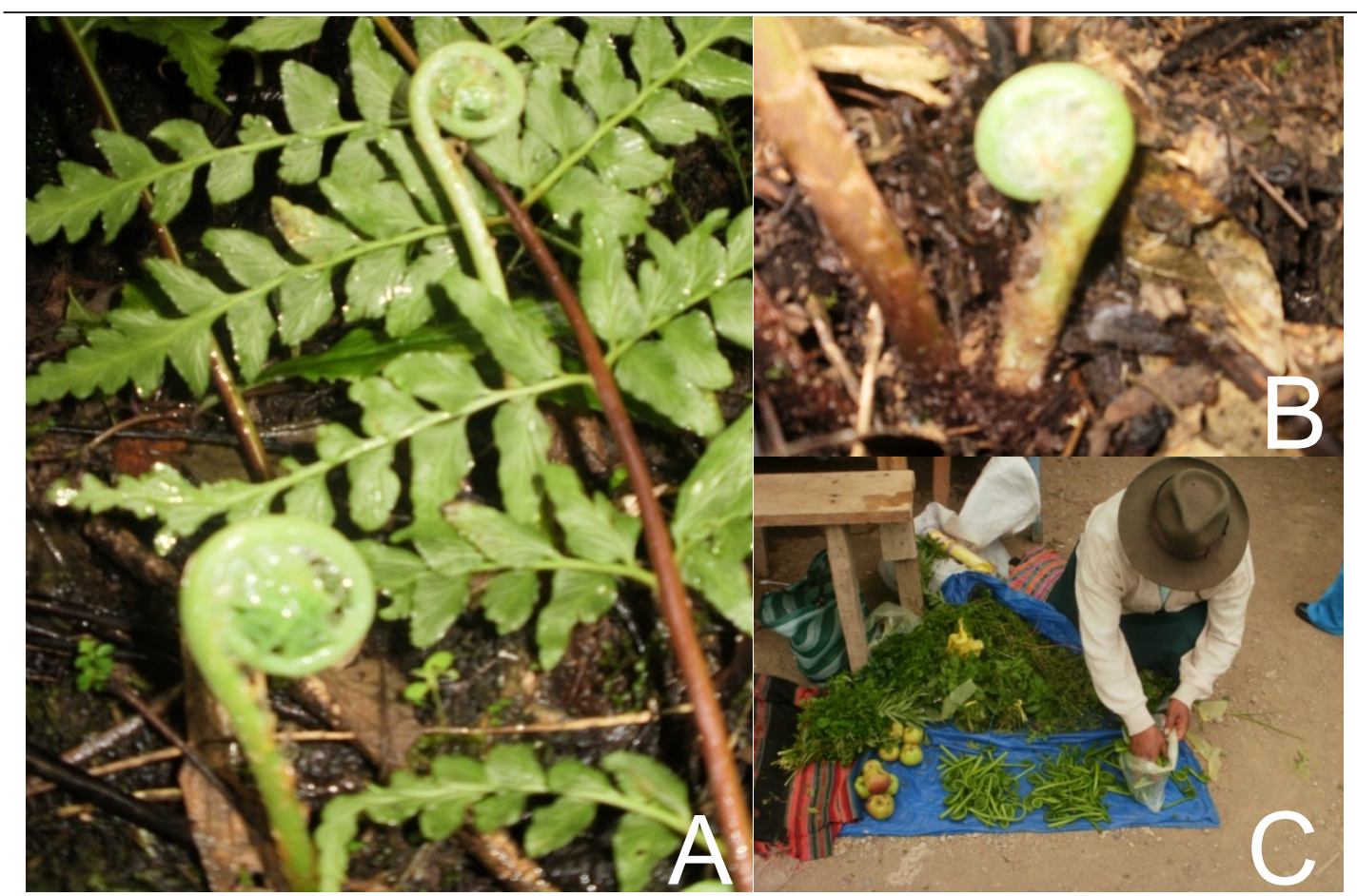

Figura 4. A. Frondas circinadas del "Ullpu" Asplenium s. L. B. Brote del "Ullpu" en el Santuario Nacional de Ampay y C. Expendio de Ullpu en la feria dominical de la ciudad de Abancay.

-aumento en el tamaño de la célula, en este proceso los brotes requieren mucha agua y casi todo el azúcar que las hojas producen. El crecimiento de los brotes es afectado principalmente por cuatro factores: la luz, el agua, la temperatura y los nutrientes el suelo (Courtis, 2014).

El agua de precipitación pluvial es esencial, así como nutrientes del suelo, que son absorbidos por las raíces. El agua es transportada por toda la planta de manera casi continua para mantener sus procesos vitales funcionando. El agua también permite que las raíces se muevan en el suelo y actúa como solvente para los minerales e hidratos de carbono. El helecho Asplenium s. L., crece en suelos húmedos y oscuros característico tierra de bosque que, tienen la propiedad de retener agua y contiene alto porcentaje de materia- -orgánica y los nutrientes (Velarde, 1991): pH: 4.6, CE: 0.1 \% \%O: 8.8, P (ppm): 1.4 y K (ppm): 300

La época de mayor producción del "Ullpu" corresponde a los meses de enero y febrero de cada año, durante esta temporada se cosechan los cayados de la especie. Los cayados de Asplenium s. L., "Ullpu" son utilizados como alimento en una forma similar a la del espárrago (Pérez, 2002) citado por (Navarrete, 2006). La calidad del sabor y delicadeza de su textura nos ha generado interés en examinar el valor alimentario con fines culinarios. Los habitantes de bajos recursos económicos que viven en las cercanías del bosque de Ampay, desde hace muchos años han incorporado en su dieta alimentaria, los brotes de renuevo son recogidos en costalillos para ser transportados hasta la feria dominical de la ciudad de Abancay. 
El Ullpu es una hortaliza nativa que crece en estado silvestre en la ladera del Ampay. Es particularmente interesante observar desde el punto de vista de uso alimentario, la diversidad de potajes que pueden ofrecer a los consumidores, por su rico valor en fibras asimilables y nutrientes que son indispensables para el mantenimiento de la salud de las personas. Desde hace tiempo la especie soporta fuertes presiones que lindan con la depredación, la excesiva saca estaría provocando la reducción poblacional de la planta y su rendimiento. No se conocen las técnicas de propagación por espora o por el rizoma, los brotes circinados podrían ser utilizados en la industria alimentaria para obtener encurtidos como alternativa de consumo humano.

\section{Literatura citada}

Akirov, I. (2009). Los Pteridofitos del Monte Zerpa Mérida - Venezuela. Universidad de Los Andes, Facultad de Ciencias Forestales y Ambientales. Pittieria 33: 79-88.

Courtis A. (2014). Crecimiento y Desarrollo Celular. Fisiología Vegetal. Facultad de Ciencias Exactas y Naturales y Agrimensura. Universidad Nacional del Nordeste. Guía. Argentina

Ganem, M. A., M. A. Morbel, I. G. E. Giudice, I. G. Michelena \& M. R. Piñeiro. (2001). Las esporas del grupo Asplenium squamosum. Polen 11:51-58.

Ganem, M. A., G. E. Giudice, M. L. \& De la Sota E. R. (2007). Revision of the group "Asplenium squamosum" in America.Candollea 62: 149-156. In Spanish, English and French abstracts.

Ganem, M. A.1, Giudice, G. E.2, Martínez, O. G.3 \& De la Sota, E. R. (2008). Asplenlacea Mett. ex A.B. Frank. Facultad de Ciencias Naturales Universidad Nacional de Salta.

Hostning, R. y Palomino C. (1997). El Santuario Nacional del Ampay: Refugio de la Intimpa en Apurímac-Perú. $1^{\circ}$ Edición.

Hassler, M. y Brian S. (2002). Familia Aspleniaceae, género Asplenium lista de especies en el mundo.
Navarrete, H. (2001). Helechos comunes de la amazonia baja ecuatoriana. Editorial Simbioe, Quito. 152 p.

Pérez, C. D. (2002). Helechos alimenticios desde los antiguos peruanos. pp. 44 Universidad Nacional de la Amazonía Peruana, Iquitos.

Pérez C. D. y Arce C. R. (2014). "ULLPU” Helechos Alimenticios desde los Antiguos peruanos. Universidad Nacional de San Antonio Abad del Cusco. Primera Edición. Cusco - Perú

Sehnem, A. (1968). Aspleniáceas. In: REITZ, R., Fl. Il. Catarin. ASPL.

Tryon, A. \& R.Tryon. (1982). Ferns and allied Plants, with Special Reference Tropical America. Harvard University. Habit Photography principally by Walter H. Hodge. Springer-Verlag, NewYork lnc.

Tryon, R. \& R. Stolze. (1993). Aspleniaceae 21. Polypodiaceae Pteridophyta of Perú. Fieldiana, Bot. 18:26-32.

Venero, G. \& Tupayachi, H. (1989). Inventario de Flora del Ampay. Boletín de Lima $\mathrm{N}^{\circ} 61$.

Velarde, Andrade Luz M. (1991). Taxonomía y composición florística de los pteridofitos del Santuario Histórico de Machupicchu. Tesis. Cusco. 